\title{
Intención emprendedora: percepción desde los estudiantes universitarios
}

\section{Entrepreneurial intention: perception from university students}

\author{
Helen Lloja Rengifo ${ }^{1, a}$ 0000-0002-0882-4034 \\ Emith Sarai Chugnas Malimba ${ }^{1, \mathrm{~b}}$ 0000-0003-2158-9283 \\ Zoilita Tello Fasanando ${ }^{1, \mathrm{c}} 0000-0003-4082-2255$ \\ Jose Joel Cruz Tarrillo ${ }^{1, d}$ 0000-0002-6372-5055 \\ ${ }^{1}$ Universidad Peruana Unión, Perú. \\ ahelenlloja@upeu.edu.pe \\ bemithchugnas@upeu.edu.pe \\ czoilitatello@upeu.edu.pe \\ diose.cruz@upeu.edu.pe
}

Recibido el: 08/09/2021

Aceptado el: 05/11/2021

Publicado el: 15/12/2021

\section{Resumen}

La presente investigación tiene como objetivo determinar los factores de mayor predominancia en la intención emprendedora. Fue realizada desde el enfoque cuantitativo, con un diseño no experimental de tipo descriptivo. Se llevó a cabo en universidades de la ciudad de Tarapoto, región San Martín, Perú. Los participantes fueron 297 estudiantes. Asimismo, los datos fueron recolectados a través de encuestas virtuales por medio de la aplicación Google Forms, para lo cual se utilizó un instrumento que fue validado por expertos y se comprobó su fiabilidad a través de Alpha de Cronbach. Se obtuvo un coeficiente de 0.95 . Los resultados dieron a conocer que el factor personal se encuentra posicionado en un nivel alto con un $68 \%$ y el factor recursos en un nivel medio con $54 \%$, los cuales son los más predominantes para fomentar una intención emprendedora en los universitarios; pero aun así se considera que se necesita un mayor compromiso e interés por parte del factor recursos. En conclusión, se puede notar la gran predominancia del factor personal hacia la intención emprendedora de los universitarios; de igual forma, tanto la actitud, habilidades de negocio, destrezas como las capacidades de identificar, planificar, implementar y convertir problemas en oportunidades son elementos esenciales para tener emprendedores exitosos.

Palabras clave: Intención emprendedora, emprendimiento, estudiantes universitarios.

\begin{abstract}
The objective of this research is to determine the factors that have the greatest predominance in entrepreneurial intention; It was carried out under the quantitative approach, with a descriptive non-experimental design. It was carried out at universities in the city of Tarapoto, San Martín Region, Peru. The participants were 297 students. The data was collected through virtual surveys through the Google forms application; For this, an instrument was used that was validated by experts and its reliability was verified through Cronbach's Alpha, obtaining a coefficient of 0.95 . The results revealed that the personal factor is positioned at a high level with $68 \%$ and the resource factor at a medium level with $54 \%$, these being the most predominant in promoting an entrepreneurial intention in university students, but even so, considers that a greater commitment and interest is needed on the part of the resource factor. In conclusion, we can note the great predominance of the personal factor towards the entrepreneurial intention of the university students, it is revealed that the attitude, business skills, skills; Abilities to identify, plan, implement, and turn problems into opportunities are essential elements for successful entrepreneurs.
\end{abstract}

Keywords: Entrepreneurial intention, entrepreneurship, university students. 


\section{Introducción}

Durante las últimas dos décadas, el desarrollo del emprendimiento ha influido en el crecimiento de la economía (Powers \& McDougall, 2005), lo que ha evidenciado el sacrificio que realizan los emprendedores al producir bienes y servicios que contribuyen al incremento eficiente de la productividad (Galindo \& Méndez, 2015). Debido a esto ha despertado el interés de las universidades en diseñar programas empresariales enfocados hacia el emprendimiento (Raposo \& Paço, 2011), con lo cual se fomenta el aprendizaje para una mejor preparación de cada estudiante mediante una variedad de contextos prácticos que ayudarán al momento de realizar un emprendimiento (Alvarez et al., 2006).

En relación con esto, García et al. (2019) sostuvieron que el emprendimiento es el surgimiento de un proceso conformado por acciones de diferentes ámbitos de gestión para consolidar una idea de negocio, razón por la cual es un tema indispensable que se debe llevar a cabo en el contexto académico (Alfaro et al., 2016). Desde una perspectiva más amplia sobre dicho concepto, como señalaron Valencia et al. (2013), las universidades deben fomentar el desarrollo de las habilidades personales de sus estudiantes como capacidades, actitudes, liderazgo, responsabilidad $\mathrm{y}$ trabajo en equipo; formando una actitud emprendedora (Aparicio et al., 2021). Por ello, se reconoce que el estudiante da más valor al conjunto de conocimientos específicos, puesto que tiene impacto directo y positivo en la intención de convertirse en emprendedores.

Sin duda, la decisión de formalizar un negocio nace con la intención de emprender a causa de una influencia positiva de la educación, mediante la cual se logra formar estudiantes competentes con autoeficacia, actitud y capacidad (Hassan et al., 2020). Ellos ponen en práctica lo aprendido y obtienen el mayor provecho de su creatividad e inteligencia. Así, son capaces de enfrentar las adversidades económicas (Mukhtar et al., 2021).

En la revisión de la literatura respecto a la intención emprendedora se pudo apreciar que constituye un campo reciente de investigación. Esto se debe a la falta de conocimiento sobre cómo los estudiantes convierten las intenciones en comportamientos emprendedores reales (Harima et al., 2021). Asimismo, algunos estudios realizados han discutido la influencia de la educación, como el contenido de los cursos, el modelo de enseñanza, la cultura y el ecosistema emprendedor respecto a las intenciones emprendedoras (Sansone et al., 2021).

Con el fin de dar respuesta a esta brecha de investigación nos preguntamos ¿qué factor influye en la intención de emprender del estudiante? Por esta razón se tiene el siguiente objetivo: determinar los factores que tienen mayor predominancia hacia la intención emprendedora en los estudiantes universitarios.

\section{Marco Teórico}

\section{Emprendimiento}

El término emprendimiento nace del vocablo francés entrepreneur y es entendida como una actividad en la que intervienen factores sociales, personales, culturales, políticos y económicos, que al unirse pueden influir en la intención de emprender en las personas (Durán \& Arias, 2015). Esta actividad se desarrolla a través de la creatividad, que moviliza recursos de un nivel de productividad a otro.

Según Orrego (2010), el emprendimiento es una acción particular de cada persona que opta por crear un negocio y está decidida a afrontar cualquier adversidad del entorno con tal de cumplir su objetivo. Siguiendo este primer enfoque, Acs y Amorós (2008) mencionaron que toda oportunidad descubierta considerada ventajosa despierta el interés de transformarse en emprendedor.

Desde su punto de vista, diversos autores han abordado estudios sobre el tema. Leyva et al. (2019) concibieron que el emprendimiento es el acto que tiene la persona en confiar absolutamente en su proyecto. Además, cuenta con las herramientas de preparación para sacarlo adelante, relacionadas con la creación de valor mediante el aprovechamiento de oportunidades e ideas innovadoras (López et al., 2021).

Para Gutierrez et al. (2014), en cambio, el emprendimiento no es creatividad: es crear. Dicha dinámica abarca la construcción de algo con el propósito de darle una sostenibilidad económica sin dejar de lado su calidad. También, se involucra con la pasión acompañada con herramientas esenciales como inteligencia, talento, habilidad, 
memoria. Durán y Arias (2015) concordaron que el emprendimiento es una conducta programada, enfocada hacia la creación de un negocio pequeño, donde se identifican oportunidades y riesgos que puedan intervenir en el desarrollo de sus actividades para tomar las medidas correctas y lograr el éxito deseado.

Entonces, se afirma que el desarrollo de un emprendimiento por parte de estudiantes es una iniciativa que surge en el ámbito universitario (Ortín et al., 2008), en donde la intención tiene gran influencia en el comportamiento que lo conducirá a la decisión de iniciar un negocio en el futuro (Zhang \& Huang, 2021). Cabe recalcar que el beneficio de realizar una actividad comercial se verá reflejado en el progreso económico de la sociedad, del país por intermedio de las oportunidades de empleo (Sidratulmunthah et al., 2018).

En general, se puede decir que el emprendimiento asume una actitud que nace de la idea de crear un negocio sostenible para obtener beneficios económicos. Asimismo, el impacto que se desarrolla en el ámbito académico ayuda a la formación y preparación del recurso humano a partir de las habilidades básicas para desenvolverse en el entorno tecnológico, social y económicamente dinámico (Castellanos et al., 2003).

\section{Intención emprendedora}

La intención es un estado psicológico que posee cada individuo, en el que expresa voluntad y motivación referente a un objetivo en particular (Awwad \& Al-Aseer, 2021). Es decir, influye directamente en la manera de actuar que lleva a la decisión para ejecutar una determinada acción o comportamiento pronosticado en el futuro (Atiya \& Osman, 2021) en donde también intervienen el esfuerzo y compromiso personal (Brännback \& Carsrud, 2009).

En efecto, la intención es un impulso psicológico que conduce a comportarnos de una determinada forma y ayuda en la búsqueda de oportunidades, brindando una idea más clara de lo que se deberá realizar (Krueger et al., 2000).

Por consiguiente, la intención emprendedora hace que las personas se encuentren preparadas para adoptar una iniciativa (Bird, 1968) de crear su propio negocio o aportar valor en el interior de las organizaciones existentes (Leiva et al., 2021).
Referente a esto, Pfeifer et al. (2016) consideraron que su cumplimiento se da cuando el individuo tiene una clara intención de emprender.

En este sentido, las universidades cumplen un rol importante en el desenvolvimiento de las habilidades de los estudiantes vinculados a la intención empresarial (Hofer et al., 2013) a través de las enseñanzas impartidas en sus diferentes actividades. Por ejemplo, se realizan proyectos, clases de investigación, un estudio de mercado, publicidad, contratar el personal, desarrollo e implementación de sistemas y estructuras (Kesner \& Dalton, 1994) relacionados con el emprendimiento y creación de empresas (Sun et al., 2017). Dichas actividades ayudan a transformar las oportunidades captadas en acciones empresariales (Welter \& Baker, 2020).

De igual forma, Aparicio et al. (2021) afirmaron que el campo académico guía al comportamiento humano a través de lo percibido en la decisión de realizar acciones empresariales y además a considerarlas como una opción profesional (Lucas \& Cooper, 2004).

Por otro lado, Su et al. (2021) agregaron que los universitarios tienen más intención de emprender después de la implementación de la educación emprendedora. La razón es que el abrir una empresa requiere de una serie de habilidades y competencias diferentes, dependiendo del tipo de negocio que desee formalizar (Bakkar et al., 2021). En otras palabras es un constructo fundamental para la transformación de un empresario exitoso (Jena, 2020).

Por otro lado, académicos descubrieron que el llevar a cabo actividades como proyectos, talleres (Bacq et al., 2017), concursos, cooperativas y pasantías tienen una influencia positiva en la intención emprendedora (Sardeshmukh \& Nelson, 2011). Sin embargo, Krueger (2011) sostuvo que no basta solo con tener intención emprendedora para ser dueños de una empresa propia, debido a las grandes adversidades del entorno que se pueden presentar en el camino, lo que representa un obstáculo para llevar determinada acción (Pruett et al., 2009). Alvarez et al. (2006) dieron a conocer dos de las grandes barreras principales que manifiestan los estudiantes para la creación de una empresa: (a) la falta de capital y (b) la duda sobre su capacidad emprendedora. Esto se debe a la carencia de recursos económicos y también a la 
falta de destrezas, habilidades, y vínculo con redes profesionales (Morris et al., 2017).

No obstante, se recalca el papel fundamental que juega la universidad en los estudiantes, con base en prácticas, experiencias y cualquier aspecto que brinde ayuda para tener mejor capacidad y seguridad de cómo realizarlo, dejando a un lado los miedos y teniendo en cuenta qué medidas tomar (Tomy \& Pardede, 2020). Referente a esto, Tehseen y Haider (2021) mencionaron que no todo estudiante presenta el mismo grado de convertirse en emprendedor, ya que esto depende de la carrera que decidieron estudiar. Al respecto, se debe considerar que cada universitario tiene planes y metas diferentes en su vida que están asociadas con sus respectivas carreras.

\section{Metodología}

\section{Objetivos}

La presente investigación tuvo como objetivo determinar los factores que tienen mayor predominancia hacia la intención emprendedora en los estudiantes universitarios.

\section{Diseño y tipo de estudio}

El estudio es de enfoque cuantitativo, puesto que se utilizaron encuestas para la recopilación de datos cuantificables. Con base en ello, se realizó el análisis estadístico para derivar conclusiones de la investigación (Baena, 2017). Es de tipo descriptivo; pues se registró, analizó e interpretó los resultados obtenidos en su estado natural (Tamayo \& Tamayo, 2003). Adicionalmente, tiene un diseño no experimental, ya que se buscó analizar los datos sin manipular ninguna variable mostrándose tal y como están en la realidad (Avila, 2006).

\section{Participantes}

La muestra se seleccionó mediante la técnica de muestreo no probabilístico, en vista de que no se puede establecer la seguridad de que todos los estudiantes hayan participado en la encuesta (Otzen \& Manterola, 2017), aplicando el muestreo por conveniencia. Se contó con un total de 297 participantes de la ciudad de Tarapoto, San Martín (Perú), de universidades privadas como públicas, entre hombres y mujeres de diferentes edades. Estos pertenecen a diferentes ciclos y facultades; entre ellas, se consideró las que siguen: Salud, Educación, Turismo y Hotelería, Ingeniería y Arquitectura, y Ciencias Económicas y Administrativas.

\section{Instrumento}

El instrumento utilizado para la presente investigación tomó como base el cuestionario elaborado por Pérez (2021), el cual se consideró para la recolección de datos. Asimismo, cuenta con tres factores: (a) personal, con 10 ítems; (b) recursos, con 8 ítems, y (c) social, con 4 ítems. Se conformó por una escala de medición tipo Likert del 1 al 5 en donde 1 es totalmente en desacuerdo y 5 es totalmente de acuerdo. En total, se tuvieron 22 ítems.

Para la fiabilidad se usó el coeficiente Alpha de Cronbach mediante el cual se obtuvo un valor de $\alpha=0.95$. Por lo tanto, se considera que el cuestionario tiene valores aceptables. Además, el Alpha de Cronbach para cada uno de los factores del cuestionario fue el siguiente: (a) factor personal $\alpha=0.92$; (b) factor recursos $\alpha=0.92$ y (c) factor social $\alpha=0.86$.

Cabe mencionar que el instrumento fue sometido a validez de contenido mediante juicio de cinco expertos que cuentan con una amplia experiencia y discernimiento en la rama de la investigación. Seguidamente, fue sometido al análisis de coeficiente de la V de Aiken de los 22 ítems, cuyo resultado fue un coeficiente de 0.81 ; lo que permite inferir que el instrumento es válido y se garantiza su aplicación.

\section{Método}

En cuanto al proceso de recopilación de datos, se realizó durante el periodo de 2 semanas y 3 días respectivos. El procedimiento consistió en que una vez ya formulado el cuestionario, se compartió el enlace a través de un mensaje directo por las diferentes redes sociales como Facebook/ Messenger, Instagram y WhatsApp. Se inició por los estudiantes conocidos de la propia universidad, como compañeros y amigos de aula, quienes compartían el enlace con sus compañeros y amigos estudiantes universitarios. También, colaboraron los delegados de aula a quienes se les explicó la importancia de esta investigación y se les pidió el favor de compartir el enlace en su respectivo grupo académico de WhatsApp. 
Los datos fueron recolectados a través de la plataforma Google Forms, herramienta que dio una mayor facilidad para encuestar y obtener respuestas en un tiempo más corto. Una vez obtenida el total de 300 encuestas respondidas, estos datos pasaron a ser analizados en el paquete estadístico SPSS (v26). Primero, se consideró realizar el análisis atípico para una mayor fiabilidad de los resultados, de los cuales 3 fueron anulados por no mostrar veracidad en sus respuestas. Después de esto, los 297 datos recolectados pasaron a ser procesados para su correspondiente tabulación. Además, se realizó el cálculo de medida de Kaiser Meyer Olkin (KMO) que se encarga de comprobar el grado de relación conjunta entre las variables observadas analizando sus ítems y dimensiones (Lloret et al., 2014); el resultado fue un 0.948, lo que indica que es aceptable. También, se realizó la prueba de esfericidad de Bartlett, que presentó valores de $\mathrm{X}^{2}=4618.163, \mathrm{gl}=231, \mathrm{P}$ valor $=0.000$. Seguidamente, se realizó el análisis clúster, que sirvió para reducir la información en conglomerados homogéneos (Lin \& Chen, 2006).

\section{Resultados}

En la Tabla 1, se identifica el nivel de intención emprendedora en los estudiantes universitarios de Tarapoto, San Martin (Perú) de acuerdo con el género. Se aprecia que las mujeres en mayor porcentaje con un $38.5 \%$ se encuentran en un nivel medio, mientras que los hombres obtuvieron un mayor porcentaje $36.2 \%$ en el nivel bajo.
Tabla 1. Nivel de predominancia de la intención emprendedora en estudiantes universitarios de acuerdo con el género.

\begin{tabular}{lllllll}
\hline Género & \multicolumn{2}{l}{ Bajo } & \multicolumn{2}{l}{ Medio } & \multicolumn{2}{l}{ Alto } \\
\cline { 2 - 7 } & $\mathrm{f}$ & $\%$ & $\mathrm{f}$ & $\%$ & $\mathrm{f}$ & $\%$ \\
\hline Masculino & 51 & 36.2 & 48 & 34.0 & 42 & 29.8 \\
Femenino & 46 & 29.5 & 60 & 38.5 & 50 & 32.1 \\
Total & 97 & 32.7 & 108 & 36.4 & 92 & 31.0 \\
\hline
\end{tabular}

En la Tabla 2, para el nivel de predominancia en la intención emprendedora según el tipo de universidad, se observa que en las universidades privadas existe mayor predominancia de $33.3 \%$ en el nivel alto. En cambio, en las universidades públicas existe una mayor predominancia de 42.4 $\%$ en el nivel medio.

Tabla 2. Nivel de predominancia de la intención emprendedora en estudiantes universitarios de acuerdo al tipo de universidad.

\begin{tabular}{lllllll}
\hline \multirow{2}{*}{ Universidad } & \multicolumn{2}{c}{ Bajo } & \multicolumn{2}{c}{ Medio } & \multicolumn{3}{c}{ Alto } \\
\cline { 2 - 7 } & $\mathrm{f}$ & $\%$ & $\mathrm{f}$ & $\%$ & $\mathrm{f}$ & $\%$ \\
\hline Privada & 66 & 33 & 66 & 33 & 66 & 33 \\
Pública & 31 & 31 & 42 & 42 & 26 & 26 \\
Total & 97 & 33 & 108 & 36 & 92 & 31 \\
\hline
\end{tabular}

En la Tabla 3, se presenta el nivel de predominancia de la intención emprendedora en las diferentes facultades académicas. La Facultad de Ingeniería y Arquitectura obtuvo un mayor porcentaje: 40 $\%$ en el nivel bajo. Seguidamente, la Facultad de Ciencias Económicas y Administrativas obtuvo $40.1 \%$ en el nivel alto. Por último, en la Facultad de Turismo y Hotelería, $53.3 \%$ en el nivel bajo.

Tabla 3. Nivel de predominancia de la intención emprendedora en estudiantes universitarios según facultad académica.

\begin{tabular}{lllllll}
\hline \multirow{2}{*}{ Facultades } & \multicolumn{3}{c}{ Nivel bajo } & \multicolumn{2}{c}{ Nivel medio } & \multicolumn{2}{c}{ Nivel alto } \\
\cline { 2 - 7 } & $\mathrm{f}$ & $\%$ & $\mathrm{f}$ & $\%$ & $\mathrm{f}$ & $\%$ \\
\hline Ingeniería y Arquitectura & 24 & 40 & 20 & 33 & 16 & 27 \\
Económicas y administrativas & 33 & 24 & 49 & 36 & 55 & 40 \\
Turismo y Hotelería & 8 & 53 & 4 & 27 & 3 & 20 \\
Salud & 24 & 39 & 24 & 39 & 13 & 21 \\
Educación & 8 & 33 & 11 & 46 & 5 & 21 \\
Total & 97 & 33 & 108 & 36 & 92 & 31 \\
\hline
\end{tabular}

En la Tabla 4, se aprecia que hay una mayor predominancia hacia la intención emprendedora se muestran las 3 dimensiones: como primera dimensión al factor personal, el cual obtuvo mayor predominancia en el nivel alto $68 \%$, segunda dimensión el factor recursos, nos indica que existe una mayor predominancia con el $70 \%$ en el nivel bajo. Por último, en cuanto al factor social existe una predominancia de $54 \%$ en el nivel medio. 
Tabla 4. Nivel de predominancia de la intención emprendedora en estudiantes universitario.

\begin{tabular}{lllllll}
\hline Dimensiones & \multicolumn{2}{c}{ Bajo } & \multicolumn{2}{c}{ Medio } & \multicolumn{2}{c}{ Alto } \\
\cline { 2 - 7 } & $\mathrm{f}$ & $\%$ & $\mathrm{f}$ & $\%$ & $\mathrm{f}$ & $\%$ \\
\hline Factor personal & 22 & 7 & 74 & 25 & 201 & 68 \\
Factor recursos & 209 & 70 & 76 & 26 & 12 & 4 \\
Factor social & 85 & 29 & 161 & 54 & 51 & 17 \\
\hline
\end{tabular}

En la Tabla 5, según los resultados obtenidos, en un total de 297 encuestados, un promedio mayor de 3.70 se encuentra entre 3 y 4 . Esto indica que el $26 \%$ está en un nivel neutro en cuanto a su forma de comunicarse con las demás personas cuando se encuentran en una situación problemática; el $46 \%$ está de acuerdo, lo que evidencia que se actúa de manera asertiva ante la misma situación. Por otro lado, se obtuvo como promedio más bajo de 3.09, que indica que el $34 \%$ no está ni de acuerdo ni en desacuerdo de la efectividad de los programas del Gobierno para promover el emprendimiento y el 7 $\%$ considera que los programas son los suficientes para que tengan mayor iniciativa en emprender. Asimismo, se consideró el promedio de 3.57, lo que demuestra que $30 \%$ practica una comunicación regular y el $44 \%$ tienen buenas relaciones interpersonales. De igual forma, se resalta un bajo promedio de 3.29 , lo que evidencia que el $32 \%$ de los encuestados infiere no estar seguro de haber participado en actividades empresariales y el $36 \%$ ha participado en alguna actividad relacionada con el emprendimiento.

Tabla 5. Tabla de frecuencia.

\begin{tabular}{|c|c|c|c|c|c|c|c|c|c|}
\hline Ítems & $\begin{array}{c}\text { Totalmente en } \\
\text { desacuerdo }\end{array}$ & $\begin{array}{c}\text { En } \\
\text { desacuerdo }\end{array}$ & $\begin{array}{l}\text { Ni de acuerdo ni } \\
\text { en desacuerdo }\end{array}$ & $\begin{array}{c}\text { De } \\
\text { acuerdo }\end{array}$ & $\begin{array}{l}\text { Totalmente } \\
\text { de acuerdo }\end{array}$ & Media & DE & Asimetría & Curtosis \\
\hline P1 & $2 \%$ & $14 \%$ & $31 \%$ & $41 \%$ & $11 \%$ & 3.44 & 0.943 & -0.376 & -0.266 \\
\hline $\mathrm{P} 2$ & $3 \%$ & $18 \%$ & $34 \%$ & $36 \%$ & $9 \%$ & 3.31 & 0.961 & -0.218 & -0.477 \\
\hline P3 & $2 \%$ & $14 \%$ & $33 \%$ & $38 \%$ & $13 \%$ & 3.45 & 0.958 & -0.299 & -0.312 \\
\hline P4 & $2 \%$ & $13 \%$ & $35 \%$ & $38 \%$ & $14 \%$ & 3.49 & 0.934 & -0.241 & -0.332 \\
\hline P5 & $6 \%$ & $17 \%$ & $30 \%$ & $33 \%$ & $15 \%$ & 3.34 & 1.095 & -0.295 & -0.578 \\
\hline P6 & $3 \%$ & $11 \%$ & $32 \%$ & $41 \%$ & $13 \%$ & 3.49 & 0.956 & -0.453 & -0.044 \\
\hline P7 & $2 \%$ & $15 \%$ & $31 \%$ & $41 \%$ & $10 \%$ & 3.42 & 0.949 & -0.366 & -0.333 \\
\hline P8 & $6 \%$ & $16 \%$ & $32 \%$ & $36 \%$ & $10 \%$ & 3.29 & 1.042 & -0.386 & -0.374 \\
\hline P9 & $4 \%$ & $15 \%$ & $34 \%$ & $36 \%$ & $12 \%$ & 3.38 & 0.993 & -0.313 & -0.310 \\
\hline P10 & $3 \%$ & $11 \%$ & $30 \%$ & $42 \%$ & $13 \%$ & 3.50 & 0.970 & -0.510 & -0.036 \\
\hline P11 & $6 \%$ & $15 \%$ & $31 \%$ & $38 \%$ & $10 \%$ & 3.31 & 1.039 & -0.430 & -0.329 \\
\hline P12 & $7 \%$ & $15 \%$ & $30 \%$ & $36 \%$ & $13 \%$ & 3.32 & 1.089 & -0.417 & -0.433 \\
\hline P13 & $6 \%$ & $14 \%$ & $32 \%$ & $37 \%$ & $11 \%$ & 3.35 & 1.036 & -0.430 & -0.262 \\
\hline P14 & $6 \%$ & $16 \%$ & $29 \%$ & $38 \%$ & $11 \%$ & 3.32 & 1.070 & -0.424 & -0.434 \\
\hline P15 & $5 \%$ & $15 \%$ & $32 \%$ & $35 \%$ & $13 \%$ & 3.36 & 1.038 & -0.335 & -0.402 \\
\hline P16 & $4 \%$ & $18 \%$ & $28 \%$ & $38 \%$ & $13 \%$ & 3.39 & 1.038 & -0.335 & -0.553 \\
\hline P17 & $4 \%$ & $16 \%$ & $32 \%$ & $36 \%$ & $13 \%$ & 3.36 & 1.028 & -0.324 & -0.408 \\
\hline P18 & $8 \%$ & $21 \%$ & $34 \%$ & $30 \%$ & $7 \%$ & 3.09 & 1.054 & -0.200 & -0.569 \\
\hline P19 & $2 \%$ & $10 \%$ & $30 \%$ & $44 \%$ & $14 \%$ & 3.57 & 0.928 & -0.497 & 0.069 \\
\hline P20 & $1 \%$ & $9 \%$ & $26 \%$ & $46 \%$ & $18 \%$ & 3.70 & 0.889 & -0.428 & -0.217 \\
\hline P21 & $2 \%$ & $13 \%$ & $26 \%$ & $47 \%$ & $12 \%$ & 3.54 & 0.940 & -0.554 & -0.055 \\
\hline $\mathrm{P} 22$ & $3 \%$ & $13 \%$ & $36 \%$ & $37 \%$ & $11 \%$ & 3.40 & 0.950 & -0.321 & -0.176 \\
\hline
\end{tabular}

En la Tabla 6, se identifican tres componentes principales que han sido extraídos y cuyos evaluadores superan la unidad. Entre los tres componentes extraídos consiguen explicar un $65.343 \%$ de la varianza de los datos originales. Se puede decir que, de la totalidad del tema estudiado, el componente 1 explica dicho fenómeno en un $51.463 \%$; el componente 2 , en un $8.932 \%$ y el componente 3 en un $4.948 \%$. Sí se unen los tres componentes, se obtendrá un porcentaje de $65.343 \%$. 
Tabla 6. Varianza total explicada.

\begin{tabular}{|c|c|c|c|c|c|c|c|c|c|}
\hline \multirow[t]{2}{*}{ Componente } & \multicolumn{3}{|c|}{ Autovalores iniciales } & \multicolumn{3}{|c|}{ Sumas de cargas al cuadrado } & \multicolumn{3}{|c|}{ Sumas de cargas al cuadrado } \\
\hline & Total & $\begin{array}{c}\% \text { de } \\
\text { varianza }\end{array}$ & $\begin{array}{c}\% \\
\text { acumulado }\end{array}$ & Total & $\begin{array}{c}\% \text { de } \\
\text { varianza }\end{array}$ & $\begin{array}{c}\% \\
\text { acumulado }\end{array}$ & Total & $\begin{array}{c}\% \mathrm{de} \\
\text { varianza }\end{array}$ & $\begin{array}{c}\% \\
\text { acumulado }\end{array}$ \\
\hline 1 & 11.322 & 51.463 & 51.463 & 11.322 & 51.463 & 51.463 & 5.542 & 25.193 & 25.193 \\
\hline 2 & 1.965 & 8.932 & 60.394 & 1.965 & 8.932 & 60.394 & 5.043 & 22.921 & 48.114 \\
\hline 3 & 1.089 & 4.948 & 65.343 & 1.089 & 4.948 & 65.343 & 3.790 & 17.228 & 65.343 \\
\hline 4 & 0.755 & 3.433 & 68.776 & & & & & & \\
\hline 5 & 0.698 & 3.174 & 71.950 & & & & & & \\
\hline 6 & 0.641 & 2.916 & 74.866 & & & & & & \\
\hline 7 & 0.580 & 2.636 & 77.502 & & & & & & \\
\hline 8 & 0.561 & 2.552 & 80.054 & & & & & & \\
\hline 9 & 0.527 & 2.393 & 82.447 & & & & & & \\
\hline 10 & 0.503 & 2.285 & 84.732 & & & & & & \\
\hline 11 & 0.410 & 1.863 & 86.594 & & & & & & \\
\hline 12 & 0.394 & 1.793 & 88.387 & & & & & & \\
\hline 13 & 0.331 & 1.506 & 89.893 & & & & & & \\
\hline 14 & 0.330 & 1.500 & 91.393 & & & & & & \\
\hline 15 & 0.301 & 1.370 & 92.763 & & & & & & \\
\hline 16 & 0.287 & 1.305 & 94.067 & & & & & & \\
\hline 17 & 0.263 & 1.196 & 95.263 & & & & & & \\
\hline 18 & 0.245 & 1.112 & 96.375 & & & & & & \\
\hline 19 & 0.222 & 1.011 & 97.386 & & & & & & \\
\hline 20 & 0.211 & 0.958 & 98.344 & & & & & & \\
\hline 21 & 0.193 & 0.876 & 99.220 & & & & & & \\
\hline 22 & 0.172 & 0.780 & 100.000 & & & & & & \\
\hline
\end{tabular}

En la Tabla 7, de análisis de componentes se obtuvo tres agrupaciones exactas de acuerdo con los tres factores: (a) factor recursos, (b) factor personal y (c) factor social. Se observó que el componente 1 engloba al factor recursos, que tiene relación con aspectos referentes al servicio que brinda la universidad brindándoles mayor motivación, aprendizaje, prácticas con tal de fomentar su intención emprendedora; de igual manera, se considera que el Gobierno tiene un rol importante que desempeñar en este rubro mediante actividades que incentiven a emprender a los jóvenes, pues ellos son el futuro del país. En el componente 2, se encuentra el factor personal, donde se considera que para desempeñar un papel emprendedor es importante contar habilidades, capacidades y desarrollo de autoconfianza para los negocios. En el componente 3, se hace referencia al factor social, que explica la forma de relacionarse con los demás y de cómo se actúa en el entorno. De esta forma, se podría definir el componente 1 como "facilidades externas para el estudiante", componente 2 como "capacidades blandas y profesiones del estudiante" y componente 3 "relaciones humanas".

Tabla 7. Matriz de componente rotado.

\begin{tabular}{lr}
\hline & Componente \\
\cline { 2 - 2 } 1. Tengo las habilidades suficientes para identificar oportunidades de negocios. & 1 \\
2. Tengo habilidad para desarrollar oportunidades de negocios. & 0.822 \\
3. Continuamente observo mi entorno para identificar oportunidades de negocios. & 0.791 \\
4. Soy capaz de realizar un proyecto de negocio con los conocimientos adquiridos en mi carrera. & 0.751 \\
5. Tengo habilidades administrativas (planear, organizar, dirigir y controlar) para crear un & 0.616 \\
negocio. & 0.587 \\
6. Proyecto mi idea de negocio a largo plazo. & 0.565 \\
7. Tengo habilidades para transformar los problemas en oportunidades. & 0.643 \\
8. He participado en actividades dirigidas a fomentar el espíritu emprendedor como pláticas, & 0.583 \\
seminarios, ferias empresariales y visitas a empresas. & 0.487 \\
9. Parte de mi tiempo libre lo utilizaría para desarrollar un proyecto de negocio. & 0.552 \\
10. Tengo habilidades necesarias para ser un líder. & 0.789 \\
11. La universidad apoya a los alumnos para realizar actividades emprendedoras. & 0.729
\end{tabular}


13. Los profesores me motivan para desarrollar mi espíritu emprendedor.

14. La universidad tiene convenios con organizaciones relacionadas con el desarrollo del

15. En mi carrera existen materias que fomentan el espíritu emprendedor

16. En la universidad existen lugares para que se realicen talleres, seminarios, charlas o actividades relacionadas con el emprendimiento.

17. En mi universidad se realizan talleres, seminarios y otras actividades para fomentar el espíritu emprendedor.

18. Los programas de Gobierno son suficientes para promover el emprendimiento.

19. Mi comunicación con los demás es buena.

20. Defiendo mis puntos de vista respetando a los demás cuando estoy en una discusión.

En la Figura 1, mediante la técnica de análisis de componentes principales se han clasificado tres grupos respecto a la intención emprendedora. Estos son los que siguen: (a) factor recursos, que corresponde a clúster 1; (b) factor personal, que corresponde a clúster 2, y (c) factor social, correspondiente a clúster 3 . A continuación, se explicará cada uno de estos.

\section{Clúster 1: factor recursos}

Presenta una fuerte influencia hacia intención emprendedora mostrando que los servicios de apoyo que recibe el estudiante relacionados con el emprendimiento $3 / 4$ la experiencia de los profesores, programa de estudios, convenios, participación del Gobierno $3 / 4$ incrementan su espíritu emprendedor.

\section{Clúster 2: factor personal}

Presenta una fuerte influencia hacia la intención emprendedora revelando la importancia de contar actitudes y habilidades para identificar y desarrollar oportunidades de negocios.

\section{Clúster 3: factor social}

Presenta poca influencia hacia la intención emprendedora, puesto que la comunicación que ejercen los estudiantes en relación con sus compañeros universitarios y a las opiniones que estos tengan acerca de su iniciativa emprendedora no son importantes.

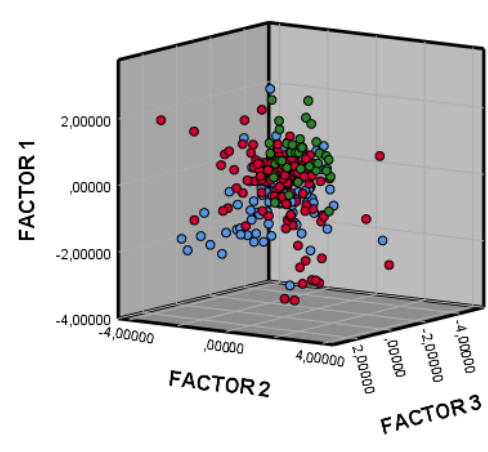

Figura 1. Análisis de clúster

\section{Discusión}

El procesamiento y análisis de datos del trabajo de investigación tiene como objetivo definir los factores que determinan la intención emprendedora en estudiantes universitarios, utilizando una metodología cuantitativa para surespectivo análisis. La aplicación empírica empleó una muestra de 297 estudiantes universitarios recolectada en el año 2021 de la ciudad de Tarapoto, San Martín (Perú).

Se determinó que el factor personal influye indiscriminadamente en la intención emprendedora en los universitarios, y que lo más importante son aquellas destrezas, habilidades y capacidades que poseen y la desarrollan de manera óptima para ejecutar un negocio. Resultados similares se obtuvieron en la investigación de Aparicio et al. 
(2021), quienes rescataron la importancia de los conocimientos y habilidades personales que deben tener los estudiantes para iniciar un negocio. También, se encontró el valor fundamental de la autoconfianza que debe estar implantada en los universitarios, la cual constituye una fortaleza de orientación y apoyo para una buena actitud y resistencia orientadas a desarrollar la intención emprendedora. De forma similar, estos resultados son consistentes con Sastre (2013). Para el investigador, la motivación intrínseca (perseverancia, optimismo y confianza) fue identificada como los factores claves para dar inicio e impulsar al emprendedor, y se sugiere que las destrezas, capacidades, habilidades propias de cada estudiante fomentan su intención emprendedora. Esto se debe a que sienten seguridad de poder lograrlo y se sienten preparados para manejar situaciones críticas que se puedan dar. Para Kautonen et al. (2015), si el estudiante siente que tiene ciertas habilidades de negocio, su intención emprendedora aumenta.

Aparte, se encontró que las habilidades personales no son suficientes para tomar una acción empresarial, aunque estas sean indispensables. El motivo es que se requiere de factores externos; por ejemplo, el factor recursos hace referencia al apoyo que brindan las instituciones como talleres, cursos, seminarios, charlas o actividades relacionadas con el emprendimiento. Los resultados de este estudio están en línea con Fiore et al. (2019), quienes expusieron acerca de aquellas facilidades y apoyo que brinda la universidad y Estado con tal de que se tenga una mejor preparación e impulso para emprender. Es así como la universidad y el Estado tienen que estar comprometidos en promover la intención emprendedora (IE) en el ámbito académico y vida diaria. De acuerdo con Sansone et al. (2021), las universidades y el Estado tiene responsabilidad en la transformación de emprendedores estudiantes por medio de diferentes actividades de estudio que fomenten su intención emprendedora; a saber, la interacción entre universidades, incubadoras, los centros de investigación sobre emprendimiento, entre otros que puedan desarrollar.

En contraste, la intención empresarial en universitarios se encuentra bastante definida por ambos factores. Por un lado, depende de las habilidades que tiene cada estudiante para tener autoconfianza en sí mismo de lograr su objetivo. Por otro, todo recurso que brindan las universidades respecto al emprendimiento ayuda significativamente mediante el aprendizaje y experiencias vividas que le servirán al momento de crear un negocio (Pham et al., 2021).

\section{Conclusiones}

Se nota la predominancia de dos factores que contribuyen al desenvolvimiento eficaz de la intención emprendedora de los universitarios. El primer factor personal da a conocer que la actitud, habilidades de negocio, destrezas, las capacidades de identificar, planificar, implementar y convertir problemas en oportunidades son elementos esenciales para tener emprendedores exitosos, que ejecuten un excelente liderazgo para garantizar la sostenibilidad y éxito. Asimismo, la predominancia del segundo factor recursos se ve reflejada por los soportes que brindan las universidades mediante charlas, cursos, talleres y desarrollo de proyectos. Esto ayuda a tener un amplio conocimiento, preparación, motivación y genera mayor seguridad para saber qué hacer y cómo hacer al momento que los estudiantes decidan enfrentarse a este gran desafío de emprender.

El entorno universitario tiene una gran responsabilidad en fomentar actitudes y comportamientos positivos en los estudiantes hacia la intención emprendedora. Es por eso que se ve necesario implementar charlas, cursos, clases, talleres que sean desarrollados de la manera óptima, eficaz e innovadora para despertar un mayor interés y motivación con el fin de desarrollar una actividad emprendedora, con estudiantes preparados para una realidad llena de cambios y desafíos. La razón es que en este entorno tienen la oportunidad de despertar directamente el espíritu emprendedor en sus estudiantes.

Del mismo modo, se requiere la intervención del Gobierno expresada en una mayor participación y compromiso en el desarrollo e implementación de nuevas actividades para apoyar a los universitarios en hacer realidad su emprendimiento. De esa manera, se contribuye con la intención emprendedora en los estudiantes.

\section{Referencias bibliográficas}

Acs, Z. J., \& Amorós, J. E. (2008). Entrepreneurship and competitiveness dynamics in Latin America. Small Business Economics, 31(3), 305-322. 10.1007/s11187-008-9133-y 
Alfaro, C. M., Cruzate, M. E., Santana, F. M., \& Peña, D. D. (2016). Caracterización de la formación en emprendimiento, una alternativa para el crecimiento económico del Municipio de Plato, Magdalena. Escenarios, 14(2), 86. 10.15665/esc.v14i2.934

Alvarez, R. D., DeNoble, A. F., \& Jung, D. (2006). Educational curricula and selfefficacy: Entrepreneurial orientation and new venture intentions among university students in Mexico. International Research in the Business Disciplines, 5, 379-403. 10.1016/ $\underline{\text { S1074-7877(06)05019-7 }}$

Aparicio, S., Urbano, D., \& Stenholm, P. (2021). Attracting the entrepreneurial potential: A multilevel institutional approach. Technological Forecasting and Social Change, 168, 120748. 10.1016/j.techfore.2021.120748

Atiya, T., \& Osman, Z. (2021). The effect of entrepreneurial characteristics on the entrepreneurial intention of university students in Oman and Sudan. Entrepreneurship and Sustainability Issues, 8(4), 217-234. 10.9770/ jesi.2021.8.4(12)

Avila, H. L. (2006). Introducción a la metodologia de la investigación (edición digital). Eumed. www.eumed.net/libros/2006c/203/

Awwad, M. S., \& Al-Aseer, R. M. N. (2021). Big five personality traits impact on entrepreneurial intention: the mediating role of entrepreneurial alertness. Asia Pacific Journal of Innovation and Entrepreneurship, 15(1), 87-100. 10.1108/apjie-09-2020-0136

Bacq, S., Ofstein, L. F., Kickul, J. R., \& Gundry, L. K. (2017). Perceived entrepreneurial munificence and entrepreneurial intentions: A social cognitive perspective. International Small Business Journal: Researching Entrepreneurship, 35(5), 639-659. $\underline{10.1177 / 0266242616658943}$

Baena, G. (2017). Metodología de la investigación (3. ${ }^{a}$ ed.). Grupo Editorial Patria. http://www. biblioteca.cij.gob.mx/Archivos/Materiales de consulta/Drogas de Abuso/Articulos/ metodologiadelainvestigacion.pdf
Bakkar, Y., Durst, S., \& Gerstlberger, W. (2021). The impact of institutional dimensions on entrepreneurial intentions of studentsInternational Evidence. Journal of Risk and Financial Management, 14(4), 174. 10.3390/ jrfm14040174

Bird, B. (1968). Implementing Entrepreneurial Ideas; The Case for Intention. Academy of Management Review, 13(3), 442-453. $\underline{10.2307 / 258091}$

Brännback, M., \& Carsrud, A. (2009). Cognitive maps in entrepreneurship: Researching sense making and action. En A. Carsrud \& M. Brännback (Eds.), Understanding the Entrepreneurial Mind (75-96). Springer. $\underline{10.1007 / 978-1-4419-0443-0}$

Castellanos, O. F., Chávez, R. D., \& Jiménez, C. N. (2003). Propuesta de formación en liderazgo y emprendimiento. Revista Innovar Journal, 1(22), 145-156. https://revistas.unal.edu.co/ index.php/innovar/article/view/25022/25553

Durán, E., \& Arias, D. (2015). Intención emprendedora en estudiantes universitarios: integración de factores cognitivos $\mathrm{y}$ socio-personales. Revista Colombiana de Ciencias Sociales, 6(2), 320-340. $\underline{10.21501 / 22161201.1528}$

Fiore, E., Sansone, G., \& Paolucci, E. (2019). Entrepreneurship education in a multidisciplinary environment: Evidence from an entrepreneurship programme held in Turin. Administrative Sciences, 9(1), 28. $\underline{10.3390 / \mathrm{admsci} 9010028}$

Galindo, M. A., \& Méndez, M. T. (2015). La actividad emprendedora y competitividad: factores que inciden sobre los emprendedores. REVESCO. Revista de Estudios Cooperativos, $121,7-32 . \underline{10.5209 / \mathrm{rev}}$

García, J. C., Villaseca, D., \& González, S. (2019). Emprendimiento femenino y financiación social: un estudio comparado. Revista de Estudios Cooperativos, 132(132), 97-121. 10.5209/REVE.65974.1.

Gutierrez, J., Asprilla, E., \& Gutiérrez, J. (2014). Emprendimiento e investigación en la escala 
de la formación profesional y la innovación empresarial en Colombia. Revista Escuela de Administracion de Negocios, O(76), 144-157. 10.21158/01208160.n76.2014.802

Harima, A., Gießelmann, J., Göttsch, V., \& Schlichting, L. (2021). Entrepreneurship? Let us do it later: Procrastination in the intentionbehavior gap of student entrepreneurship. International Journal of Entrepreneurial Behavior \& Research, 27(5), 1189-1213. 10.1108/ijebr-09-2020-0665

Hassan, A., Saleem, I., Anwar, I., \& Hussain, S. A. (2020). Entrepreneurial intention of Indian university students: The role of opportunity recognition and entrepreneurship education. Education and Training, 62(7-8), 843-861. 10.1108/ET-02-2020-0033

Hofer, A. R., Potter, J., Redford, D., \& Stolt, J. (2013). Promoting successful graduate entrepreneurship at the Technical University. OECDIlibrary,3-22. 10.1787/5k4877203bjhen

Jena, R. (2020). Measuring the impact of business management Student's attitude towards entrepreneurship education on entrepreneurial intention: A case study. Computers in Human Behavior, 107, 106275. 10.1016/j. chb.2020.106275

Kautonen, T., Van Gelderen, M., \& Fink, M. (2015). Robustness of the theory of planned behavior in predicting entrepreneurial intentions and actions. Entrepreneurship: Theory and Practice, 39(3), 655-674. 10.1111/etap.12056

Kesner, I., \& Dalton, D. (1994). Top management turnover and ceo succession: an investigation of the effects of turnover on performance. Revista de Estudios de Gestión, 31, 701-713. 10.1111/j.1467-6486.1994.tb00635.x

Krueger, N. F. (2011). Entrepreneurial resilience: Real \& perceived barriers to implementing entrepreneurial intentions. SSRN Electronic Journal, 1-9. 10.2139/ssrn.1155269

Krueger, N. F., Reilly, M. D., \& Carsrud, A. L. (2000). Competing models of entrepreneurial intentions. Journal of Business Venturing, 15(5), 411-432. 10.1016/S0883-

\section{6(98)00033-0}

Leiva,J.C.,Mora, R., Krauss-Delorme, C., Bonomo, A., \& Solís, M. (2021). Entrepreneurial intention among Latin American university students. Academia Revista Latinoamericana de Administración, 34(3), 399-418. 10.1108/ arla-05-2020-0106

Leyva, A. B., Alcántara, J. R., Espejel, J. E., \& Coronado, M. A. (2019). Formación del perfil emprendedor en educación superior en la Universidad de Sonora, México. Revista Escuela de Administración de Negocios, 86, 115-132. 10.21158/01208160.n86.2019.2293

Lin, G. F., \& Chen, L. H. (2006). Identification of homogeneous regions for regional frequency analysis using the self-organizing map. Journal of Hydrology, 324(1-4), 1-9. 10.1016/j.jhydrol.2005.09.009

Lloret, S., Ferreres, A., Hernánde, A., \& Tomás, I. (2014). El análisis factorial exploratorio de los ítems: Una guía práctica, revisada y actualizada. Anales de Psicología, 30(3), 1151-1169. 10.6018/analesps.30.3.199361

López, M. I., Rubio, S., Díaz, E., \& Martín, G. (2021). Perfil emprendedor de estudiantes universitarios: un modelo predictivo. Revista de Educación, 392, 11-33. 10.4438/1988592X-RE-2021-392-477

Lucas, W. A., \& Cooper, S. Y. (2004). Enhancing self-efficacy to enable entrepreneurship: The case of CMI's connections. SSRN Electronic Journal. 10.2139/ssrn.568383

Morris, M. H., Tsukanova, T., \& Shirokova, G. (2017). Student entrepreneurship and the university ecosystem: a multicountry empirical exploration. European J. of International Management, 11(1), 65. $\underline{\text { 10.1504/ejim.2017.10001679 }}$

Mukhtar, S., Wardana, L. W., Wibowo, A., \& Narmaditya, B. S. (2021). Does entrepreneurship education and culture promote students' entrepreneurial intention? The mediating role of entrepreneurial mindset. Cogent Education, 8(1), 1918849. 10.1080/2331186X.2021.1918849 
Orrego, C. (2010). La fenomenología y el emprendimiento. Revista Cientifica Pensamiento Y Gestión, 83, 27. https:// rcientificas.uninorte.edu.co/index.php/ pensamiento/article/view/848

Ortín, P., Salas, V., Trujillo, M. V., \& Vendrell, F. (2008). La creación de spin-off universitarias en España: características, determinantes y resultados. Economía Industrial, 368, 79-95. https://www.mincotur.gob.es/Publicaciones/ Publicacionesperiodicas/EconomiaIndustrial/ RevistaEconomiaIndustrial/368/79.pdf

Otzen, T., \& Manterola, C. (2017). Técnicas de muestreo sobre una población a estudio. International Journal of Morphology, $35(1), \quad 227-232 . \quad 10.4067 / \mathrm{S} 0717-$ $\underline{95022017000100037}$

Pérez, A. G. (2021). El emprendimiento en estudiantes universitarios: un análisis en las ciencias económicas y administrativas. Revista Inclusiones: Revista de Humanidades y Ciencias Sociales, 8(9), 204-217. https:// revistainclusiones.org/index.php/inclu/ article/view/1127

Pfeifer, S., Sarlija, N., \& Zekic, M. (2016). Shaping the entrepreneurial mindset: Entrepreneurial intentions of business students in Croatia. Journal of Small Business Management, 54(1), 102-117. 10.1111/jsbm.12133

280 Pham, D., Jones, P., Dobson, S., Liñán, F., \& Viala, C. (2021). Entrepreneurial implementation intention as a tool to moderate the stability of entrepreneurial goal intention: A sensemaking approach. Journal of Business Research, 123, 97-105. 10.1016/j.jbusres.2020.09.051

Powers, J., \& McDougall, P. (2005). University start-up formation and technology licensing with firms that go public: A resource-based view of academic entrepreneurship. Journal of Business Venturing, 20(3), 291-311. 10.1016/j.jbusvent.2003.12.008

Pruett, M., Shinnar,R., Toney, B.,Llopis, F., \&Fox, J. (2009). Explaining entrepreneurial intentions of university students: A cross-cultural study. International Journal of Entrepreneurial Behaviour and Research, 15(6), 571-594. $\underline{10.1108 / 13552550910995443}$
Raposo, M., \& Paço, A. (2011). Entrepreneurship education: Relationship between education. Psicothema, 23(3), 453-457. http://www. psicothema.com/pdf/3909.pdf

Sansone, G., Ughetto, E., \& Landoni, P. (2021). Entrepreneurial intention: An analysis of the role of student-led entrepreneurial organizations. Journal of International Entrepreneurship, 19, 399-433. 10.1007/ s10843-021-00288-6

Sardeshmukh, S., \& Nelson, R. (2011). Educating for an entrepreneurial career: Developing opportunity-recognition ability. Australian Journal of Career Development, 20(3), 4755. 10.1177/103841621102000308

Sastre, R. (2013). La motivación emprendedora y los factores que contribuyen con el éxito del emprendimiento. Ciencias Administrativas, 1, 1-10. $\quad$ https://www.redalyc.org/ pdf $/ 5116 / 511651377005 . p d f$

Sidratulmunthah, Hussain, S., \& Imran, M. (2018). Towards nurturing the entrepreneurial intentions of neglected female business students of Pakistan through proactive personality, self-efficacy and university support factors. Asia Pacific Journal of Innovation and Entrepreneurship, 12(3), 363-378. 10.1108/apjie-03-2018-0015

Su, Y., Zhu, Z., Chen, J., Jin, Y., Wang, T., Lin, C. L., \& Xu, D. (2021). Factors influencing entrepreneurial intention of university students in china: Integrating the perceived university support and theory of planned behavior. Sustainability (Switzerland), 13(8). $\underline{10.3390 / \mathrm{su} 13084519}$

Sun, H., Lo, C. T., Liang, B., \& Wong, Y. L. (2017). The impact of entrepreneurial education on entrepreneurial intention of engineering students in Hong Kong. Management Decision, 55(7), 1371-1393. 10.1108/MD06-2016-0392

Tamayo \& Tamayo, M. (2003). El proceso de la investigación cientifica. Limusa. https:// cucjonline.com/biblioteca/files/original/ 874e481a4235e3e6a8e3e4380d7adb1c.pdf 
Tehseen, S., \& Haider, S. A. (2021). Impact of universities' partnerships on students' sustainable entrepreneurship intentions: A comparative study. Sustainability, 13(9), 5025. 10.3390/su13095025

Tomy, S., \& Pardede, E. (2020). An entrepreneurial intention model focussing on higher education. International Journal of Entrepreneurial Behaviour and Research, 26(7), 1423-1447. 10.1108/IJEBR-06-2019-0370

Valencia, J. D., Valencia, A., \& Montaño, W. M. (2013). Percepciones de la formación para el emprendimiento en los estudiantes universitarios. Actualidades Pedagógicas, 62, 143-164. 10.19052/ap.2418
Welter, F., \& Baker, T. (2020). Moving contexts onto new roads: Clues from other disciplines. Entrepreneurship: Theory and Practice, 0, $1-22 . \underline{10.1177 / 1042258720930996}$

Zhang, J., \& Huang, J. (2021). Entrepreneurial self-efficacy mediates the impact of the postpandemic entrepreneurship environment on college students' entrepreneurial intention. Frontiers in Psychology, 12, 1-9. 10.3389/ fpsyg.2021.643184 